\title{
Vitamin D confers neuroprotective effects in traumatic brain injury by activating Nrf2 signaling through an autophagy-mediated mechanism
}

\section{Changmeng Cui}

Affiliated Hospital of Jining Medical University

\section{Changshui Wang}

Affiliated Hospital of Jining Medical University

Feng Jin

Affiliated Hospital of Jining Medical University

\section{Mengqi Yang \\ Jining First People's Hospital}

\section{Lingsheng Kong}

Affiliated Hospital of Jining Medical University

\section{Wenxiu Han}

Jining First People's Hospital

Pei Jiang ( $\nabla$ jiangpeicsu@sina.com )

Jining first People's Hospital

\section{Research}

Keywords: Traumatic brain injury, Vitamin D, Autophagy, Keap-Nrf2 system

Posted Date: July 13th, 2021

DOI: https://doi.org/10.21203/rs.3.rs-362065/v2

License: (9) This work is licensed under a Creative Commons Attribution 4.0 International License.

Read Full License 


\section{Abstract}

Background: Traumatic brain injury (TBI) initiates an oxidative cascade that contributes to the delayed progressive damage, whereas autophagy is critical in maintaining homeostasis during stressful challenge. We previously demonstrated that vitamin $D(V i t D)$ shows strong neuroprotective and antioxidative properties in the animal models of TBI. Therefore, the present study aimed to further explore the potential interrelationship between oxidative stress and autophagy in the progression of TBI and therapeutic mechanism of VitD.

Methods: Neuroprotective effects of calcitriol, the active form of VitD, were examined following TBI. We further evaluated the impacts of TBI and VitD treatment on autophagic process and nuclear factor E2related factor 2 (Nrf2) signaling. To confirm the mechanism, chloroquine (CQ) treatment and Nrf2 -/mice were used to block autophagy and Nrf2 pathway, respectively.

Results: We found that treatment of calcitriol markedly ameliorated the neurological deficits and histopathological changes following TBI. The brain damage impaired autophagic flux and impeded Nrf2 signaling, the major regulator in antioxidant response, consequently leading to uncontrolled and excessive oxidative stress. Meanwhile, calcitriol promoted autophagic process and activated Nrf2 signaling as evidenced by the reduced Keap1 expression and enhanced Nrf2 translocation, thereby mitigating TBI-induced oxidative damage. To further confirm whether autophagy was responsible for Keap1 degradation and Nrf2 activation, the lysosomal inhibitor, CQ, was used to block autophagy. Our data suggested that CQ treatment abrogated calcitriol-induced autophagy and compromised Nrf2 activation with increased Keap1 accumulation and reduced expression of Nrf2-targeted genes. Additionally, both CQ treatment and Nrf2 genetic knockout abolished the protective effects of VitD against both TBI-induced neurological deficits and neuronal apoptosis.

Conclusions: Therefore, our work demonstrated a neuroprotective role of VitD in TBI by triggering Nrf2 activation, which might be mediated by autophagy.

\section{Introduction}

Traumatic brain injury (TBI) is one of the most common causes of death and long-term impairment that affects all ages (Stout et al., 2018). The brain damage is induced by both primary and secondary injury processes. The primary injury is the mechanical disruption of brain tissue that occurs immediately, whereas secondary injury subsequently develops over time via intricate processes, including excitotoxicity, neuroinflammation and oxidative stress that ultimately contribute to neuronal loss (Ma et al., 2018). Because of its complexity, effective therapies for the treatment of TBI are still inadequate.

Autophagy is a major proteolytic system that senses intracellular stressful conditions and rapidly mounts a molecular response to deal with the damage through sequestration and degradation of dysfunctional organelles and compromised proteins (Zhang et al., 2019). The autophagic process is orchestrated through a series of autophagy-related genes (ATG genes), such as LC3-II (derived from LC3-I upon 
lipidation) and Beclin-1, which are verified biomarkers for evaluating autophagy. In addition, the adaptor protein p62 serves as a cargo receptor responsible for recognizing and loading ubiquitinated proteins into autophagosomes for degradation in selective autophagy (Su et al., 2019). Notably, p62 also activates nuclear factor E2-related factor 2 (Nrf2) signaling, a well-characterized cellular defense mechanism against oxidative stress, by interacting with Kelch-like ECH-associated protein 1 (Keap1) (Saito et al., 2020). In the cytoplasm, Nrf2 is bound to its partner, Keap1, and is rapidly degraded through the ubiquitinproteasome pathway (Zheng et al., 2020). It has been demonstrated that p62 directly interacts with Keap1, thereby dissociating it from Nrf2 and directing it toward autophagic degradation (Deng et al., 2020; Dodson et al., 2015).

Vitamin D (VitD) now is recognized as a novel neuroactive steroid, which has antioxidant and neuroprotective activities in addition to its classical function in bone metabolism and calcium-phosphate homeostasis (Cui et al., 2019). We previously demonstrated that 1,25-dihydroxyvitamin $\mathrm{D}_{3}$ (calcitriol), the active form of VitD, is effective in preventing TBI-induced behavioral abnormalities and oxidative stress (Cui et al., 2017). Except that, calcitriol treatment also promotes autophagic flux, preventing autophagosome accumulation following TBI (Cui et al., 2017). Emerging evidence has indicated that the antioxidant activity of VitD is tightly related to Keap1/Nrf2 signaling (Zheng et al., 2020; Nachliely et al., 2019), suggesting that vitamin $D$ receptor (VDR) activation may mediate the interaction between autophagy and the Keap1/Nrf2 system to facilitate the neuroprotective functions of VitD.

Therefore, the present study aims to test the protective actions of VitD following TBI and its impacts on autophagy and Nrf2 signaling. Moreover, through blocking autophagic process by using chloroquine (CQ) and genetic knockout of Nrf2, we further sought to confirm the hypothesis that autophagy may interact with Keap1/Nrf2 system to contribute to the neuroprotective effects of VitD.

\section{Materials And Methods}

\section{Animal care and drug treatment}

Adult male CD1 Elite mice (22-28 g, 10-12 weeks; Jining, China) were used in this study. Nrf2 knockout $\left(\mathrm{Nrf2}^{-/-}\right)$CD1 male mice were obtained from Dr. Chunyan Li (Second Hospital of Hebei Medical Univercity). The Institutional Animal Care and Use Committee of Jining Medical University approved all the experiments, which were performed according to the guidelines of the National Institutes of Health Guide for the Care and Use of Laboratory Animals (NIH Publications No.80-23). The mice were housed under standard conditions and were randomly assigned among one of 5 groups: sham, TBI, TBI + low dose $(0.5 \mu \mathrm{g} / \mathrm{kg})$ of calcitriol, TBI + medium dose $(1 \mu \mathrm{g} / \mathrm{kg})$ of calcitriol, and TBI + high dose $(3 \mu \mathrm{g} / \mathrm{kg})$ of calcitriol. The animals received daily gavage of vehicle or different doses of calcitriol for 14 days. Moreover, to confirm the neuroprotective mechanisms of VitD, CQ (30 mg/kg/day) was co-treated with calcitriol by intraperitoneal injection to block autophagy flux.

\section{Mouse model of TBI}


A previously described (Cui囚Song 2017), a 10-mm-long midline scalp incision was made to expose the skull. TBI was induced by hitting the brain surface at the center of the craniotomy with a 2.5-mm-diameter rounded metal tip at a velocity of $4 \mathrm{~m} / \mathrm{s}$ and a deformation depth of $2 \mathrm{~mm}$ using a controlled cortical impact (CCI) device (CCI Model 6.3; Custom Design, USA). The bone flap was immediately replaced and sealed, and the scalp was sutured.

\section{Neurological score evaluation}

At 1-14 days following TBI, the neurological scores were determined as Neurological Severity Scores, a composite of motor, sensory, reflex, and balance tests (normal score: 2-3; maximal deficit score: 18; Additional file 1).

\section{The Morris water maze (MWM) test}

The apparatus consisted of a circular black water tank $(180 \mathrm{~cm}$ in diameter, $50 \mathrm{~cm}$ high) filled with water $\left(26^{\circ} \mathrm{C}\right)$ to a depth of $30 \mathrm{~cm}$. An escape platform (diameter $12 \mathrm{~cm}$, height $28 \mathrm{~cm}$, painted opaque) submerged $2 \mathrm{~cm}$ below the water surface was placed in the middle of one of the quadrants equidistant from the tank wall and the center of the pool. All the mice were trained to find the platform before the sham operation or the induction of TBI. For each trial, each mouse was randomly placed into a quadrant start point (N, S, E, or W) facing the wall of the pool and was allowed a maximum of $60 \mathrm{~s}$ to find the escape platform. The mice that failed to escape within $60 \mathrm{~s}$ were placed on the platform for a maximum of $20 \mathrm{~s}$ and returned to their cage to await a new trial (intertrial interval, $10 \mathrm{~min}$ ). Probe trials were conducted at 11-14 days following the induction of TBI or the sham operation. The time spent in the target quadrant and the swim speeds were evaluated on the last day of the test after the platform was removed.

\section{Histopathological staining}

After fixation, the brains were embedded in paraffin and sliced into 4- $\mu \mathrm{m}$ coronal sections at the level of the bregma and stained with hematoxylin and eosin (H\&E). Apoptosis was assessed using terminal deoxynucleotidyl transferase-mediated cyanine-dUTP nick-end labeling (TUNEL) following the manufacturer's protocol. Nuclei were counterstained with DAPI (Beyotime Biotechnology, China). For each group, sections from three different mice were used for quantification.

\section{Transmission electron microscopy (TEM)}

Tissues were immersed in $2 \%$ glutaraldehyde and $1 \%$ osmium tetroxide (Sigma-Aldrich; Merck KGaA) for $2 \mathrm{~h}$ at $4{ }^{\circ} \mathrm{C}$, and then dehydrated via a graded ethanol series. Following the displacement of ethanol with propylene oxide, the tissues were embedded in Epon (both from Sigma-Aldrich) and sectioned along the coronal plane with a diamond knife (FernAnclez-hlorln 1953; Ivan Sorvall, Inc., New York, NY, USA) at a thickness of $60 \mathrm{~nm}$. The sections were stained with lead citrate and observed using a CM-120 electron microscope (Philips, Eindhoven, Netherlands). In order to quantify the alteration of the number of the autolysosomes, the area of the cell cytoplasm was measured by using Image-Pro Plus 6.0. 


\section{Western blot analysis}

Proteins $(50 \mu \mathrm{g})$ were separated by $12 \%$ SDS-PAGE and transferred onto a nitrocellulose membrane. The membrane was blocked with $5 \%$ nonfat milk at room temperature for $2 \mathrm{~h}$ and then incubated overnight at $4{ }^{\circ} \mathrm{C}$ with primary antibodies against microtubule-associated protein light chain 3 (LC3) (1:500, PM036, MBL, Nagoya, Japan), p62 (1:1,000, ab101266, Abcam, Cambridge, MA, USA), beclin 1 (1:1,000, ab137161, Abcam), Keap1 (1:1,000, ab118285, Abcam), Nrf2 (1:1,000, ab62352, Abcam), beta-actin (1:1,500, A1978, Sigma, USA), and PCNA (1:500, ab92552, Abcam). The gray values of the protein bands were measured using ImageJ software and normalized to that of $\beta$-actin that was used as an internal control.

\section{Immunofluorescence staining}

Frozen cross-sections $(15 \mu \mathrm{m})$ were prepared and examined. The sections were incubated with primary antibodies against Keap1 (1:200, ab139729), p62 (1:200, ab56416), and Nrf2 (1:1,000, ab62352) (all from Abcam) overnight at $4{ }^{\circ} \mathrm{C}$, and then with a mixture of FITC- and TRITC-conjugated secondary antibodies for $2 \mathrm{~h}$ at room temperature. Images were captured under a fluorescence microscope (Olympus, Japan).

\section{Real-time PCR analysis}

Total RNA was extracted using Trizol reagent (Invitrogen, USA) following the manufacturer's instructions. Quantitative PCR was performed on a Bio-Rad Cx96 Detection System (Bio-Rad, USA) using a SYBR green PCR kit (Applied Biosystems, USA) and gene-specific primers (Additional file 2).

\section{Detection of oxidative parameters}

The generation of reactive oxygen species (ROS) was determined by fluorescence-labeled dihydroethidium (DHE). Frozen cross-sections $(15 \mu \mathrm{m})$ were incubated in DHE for $30 \mathrm{~min}$ at $37^{\circ} \mathrm{C}$ in a dark humidified chamber. The sections were rinsed three times in PBS and observed using an inverted fluorescence microscope (Olympus, Japan). Malondialdehyde (MDA) levels were measured using the thiobarbituric acid reactive substances (TBARS) assay. The activities of superoxide dismutase (SOD), catalase (CAT), and glutathione (GSH) were determined using SOD, CAT, and GSH assay kits, respectively (Nanjing Jiancheng Bioengineering Institute, China).

\section{Primary cortical neuron culture}

The skull, blood and meninges were carefully removed from fetal mouse brains. After the cortical tissue was digested in $0.25 \%$ trypsin $\left(\mathrm{BI}\right.$, Israel) for $5 \mathrm{~min}$ at $37^{\circ} \mathrm{C}$, the suspensions, containing fetal bovine serum ( $\mathrm{BI}$, Israel), were passed through filters with a $0.22-\mu \mathrm{m}$ pore size (Millipore, USA) and then centrifuged at 1,500 rpm for $5 \mathrm{~min}$. The cells were distributed in poly-D-lysine-coated plates. The medium was replaced with neurobasal medium supplemented with streptomycin, penicillin, HEPES, glutamate, and B27 (BI, Israel). The cells were exposed to different doses of calcitriol (1nM, 10nM, 100nM, 500nM) 
for $24 \mathrm{~h}$. The cells were pre-treated for $6 \mathrm{~h}$ with $25 \mu \mathrm{M}$ CQ and then treated with the indicated doses of calcitriol.

\section{Cell viability analysis}

MTT was added to each well of a 24-well plate followed by incubation at $37^{\circ} \mathrm{C}$ for $1 \mathrm{~h}$. The purple formazan crystals formed through the reduction of MTT were then dissolved in $500 \mu \mathrm{L}$ DMSO, and the absorbance of the wells was recorded at $590 \mathrm{~nm}$. Cell viability was calculated by the absorbance ratio of the treated group to that of the control.

\section{Autophagic flux analysis}

Autophagic flux was detected by using the RFP-GFP-LC3 adenovirus (Hanbio, China). After plating the cells in a 24-well plate at a density of $1 \times 10^{4}$ cells/ dish and incubating with mRFP-GFP-LC3 adenovirus for $24 \mathrm{~h}$. Autophagic flux was observed under an inverted fluorescent microscope (Olympus, Japan). The yellow puncta indicated autophagosomes, and the red puncta indicated autolysosomes.

\section{Statistical analysis}

The results were expressed as means \pm SD. All the analyses were performed using SPSS 17.0 software. Statistical significance was determined using one-way analysis of variance (ANOVA), and the StudentNewman-Keuls post hoc test was used to determine differences among different groups. A $P$-value < 0.05 was considered statistically significant.

\section{Results}

\section{The effects of calcitriol on TBI-induced neurological deficits}

The neurological severity scores were assessed at 1-14 days after TBI (Additional file 3). At 14 days, the level of neurological injury was significantly increased in the TBI group compared with that in shamoperated animals $(P<0.01)$. In addition, compared with the TBI-only group, all calcitriol treatment groups showed improved neurological deficit scores $(P<0.01)$. No significant differences were found among the mice treated with different calcitriol doses (Fig. 1A).

\section{The effects of calcitriol on TBI-induced learning and memory ability impairment}

The MWM hidden platform task was used to investigate whether calcitriol could improve the spatial memory deficits at 11-14 days after TBI (Additional file 4A). As shown in Fig. 1B, compared with the sham operation group, mice with TBI spent longer searching for the hidden platform at 14 days postsurgery $(P<0.01)$. In contrast, calcitriol-treated mice displayed a markedly shorter latency time compared with that in the TBI-only treatment group $(P<0.01$ for the low-dose and medium-dose groups; $P<0.05$ for 
the high-dose group). In the probe trials (Fig. 1C), mice with TBI spent less time than their sham-operated counterparts swimming toward the goal quadrant that previously contained the platform $(P<0.01)$. Nevertheless, mice in the low-dose and medium-dose calcitriol treatment groups displayed improved learned bia $(P<0.01)$. Representative traces at 14 days are depicted in Fig. 1D and 1E. No significant differences in swimming speeds were found among the groups, indicating that the observed differences were not a result of an inability to execute the swimming task (Additional file 4B).

\section{The effects of calcitriol on neuronal cell death after TBI}

As depicted in Fig. 2, the cortical neurons in the sham operation group were arranged in an orderly manner, the cytoplasm was transparent, the cell nuclei showed a round or oval shape, the chromatin was evenly distributed, and the nucleoli were clear. After TBI, the opposite morphology was observed. In the calcitriol treatment groups, the amount and level of neuronal degeneration, necrosis, and loss were significantly attenuated. Likewise, only a small number of TUNEL-positive neurons were found in the sham operation group. However, the neuronal cells in the TBI-only group were arranged in a disorderly manner, and a greater number of apoptotic neurons were observed $(P<0.01)$. After calcitriol treatment, the number of apoptotic cortical neurons was significantly decreased $(P<0.01)$.

\section{The effects of calcitriol on TBI-induced autophagy impairment}

TEM analysis demonstrated that in the TBI-only group, there was a markedly increased accumulation of autophagosomes around the nucleus than in the sham operation group $(P<0.01)$ (Fig. 3A). However, in calcitriol-treated mice, the autophagosome abundance was reduced, whereas the autolysosomes were increased ( $P<0.01$ versus the TBI-only group). Moreover, we found that $\angle C 3$ and beclin $1 \mathrm{mRNA}$ levels were unchanged after TBI, whereas that of p62 was markedly increased $(P<0.01)$. The mRNA expression levels of $L C 3$, p62, and beclin 1 were all markedly higher in the calcitriol treatment groups than in the TBIonly treatment group ( $P<0.01$ for LC3 and p62; $P<0.05$ for beclin 1$)$ (Fig. 3C). Intriguingly, TBI induced the protein expression of LC3-II and p62 $(P<0.01)$, but this effect was partly reversed following repeated calcitriol treatment $(P<0.01)$. However, no significant changes in beclin 1 protein expression were observed in the TBI-only treatment group, whereas beclin 1 was upregulated after calcitriol treatment $(P<$ 0.01) (Fig. 3D, E). In addition, as shown in Fig. 3F and Fig. 3G, blocking autophagosome-lysosome fusion with CQ did not lead to an additional increase in LC3-II protein levels in TBI-only-treated mice. These results suggested that TBI induced autophagy dysfunction and impaired autophagosome clearance, which was mitigated by calcitriol treatment.

\section{The effects of calcitriol on the Keap1-Nrf2 pathway}

As we found that calcitriol treatment could rescue TBI-induced autophagic flux dysfunction, we further investigated whether autophagic process affected the Keap1-Nrf2 pathway. The expression levels of Keap1 and Nrf2 were measured by western blotting (Fig. 4A, B). The level of Keap1 was higher in the TBIonly group than in the sham operation group $(P<0.01)$; however, calcitriol treatment partially reduced the Keap1 expression level $(P<0.01)$. Immunofluorescent staining revealed similar changes in the protein 
levels of p62 and Keap1 (Fig. 4C and Fig. 4D). TBI led to a significant reduction in the expression of Nrf2 in the nucleus $(P<0.01)$, while calcitriol treatment increased the nuclear translocation of $\mathrm{Nrf2}(P<0.01)$. The mRNA levels of Nrf2 target genes (NADPH quinone dehydrogenase 1 [Nqo1], heme oxygenase 1 [HO1], glutamate-cysteine ligase catalytic subunit [Gc/c]) were then evaluated by qPCR (Fig. 4G, H, I). In line with the expression levels of Nrf2 in the nucleus, the mRNA levels of Nq01, HO1, and Gclc were significantly decreased in the TBI-only treatment group $(P<0.01)$, whereas the expression of these genes was significantly increased in the calcitriol treatment groups $(P<0.01)$.

\section{The effects of calcitriol on TBI-induced oxidative damage}

As shown in Fig. 5, calcitriol treatment markedly enhanced the activities of the antioxidant enzymes SOD, CAT, and GSH, while also reducing lipid peroxidation product (MDA) levels and ROS production.

\section{The effects of inhibiting autophagy and knocking out Nrf2 on the neuroprotective activity of calcitriol}

Our data demonstrated that both autophagy flux and the Keap1-Nrf2 pathway were activated by calcitriol treatment. Consequently, we assessed whether a noncanonical signaling network that includes both autophagy flux and the Keap1-Nrf2 pathway is induced by calcitriol. First, mice in the calcitriol groups were treated with $\mathrm{CQ}$, a selective autophagy inhibitor that prevents autophagosome-lysosome fusion. As shown in Fig. 6, CQ treatment markedly suppressed the Keap1-Nrf2 pathway, inducing Keap1 protein expression and decreasing that of Nrf2 in the nucleus when compared with the calcitriol-only treatment groups $(P<0.01)$. The levels of Nrf2 target genes were also examined. The mRNA levels of Nqo1 and Gclc were significantly decreased with CQ treatment, whereas ROS production was significantly increased ( $P<0.01$ versus the calcitriol-only group). Interestingly, CQ treatment blocked the ameliorative effect of calcitriol on neurological behavior and suppressed neuronal apoptosis (Fig. 7), highlighting the critical role of autophagy flux in the neuroprotective effects of calcitriol. Additionally, $\mathrm{Nrf2}^{-/-}$mice exhibited lower Nqo1 and Gclc mRNA levels after calcitriol treatment $(P<0.01$ versus the calcitriol-only treatment groups). As expected, the neuroprotection effect of calcitriol on TBI was abrogated in Nrf2 knockout mice, which displayed markedly impaired neurological functions and neuronal apoptosis (Fig. 7). The neurological score evaluation and MWM hidden platform task data are depicted in Additional file 5 and 6.

\section{Effect of calcitriol on neuronal autophagy and Keap1-Nrf2 pathway in vitro}

As shown in Fig. 8A, the results from the MTT assay demonstrated that while calcitriol at concentration of $1 \mathrm{nM}$ and $10 \mathrm{nM}$ had no toxic effect, high concentration of calcitriol exposure (100nM and 500nM) significantly reduced cell viability when compared to the control group $(P<0.01)$. Therefore, the impact of calcitriol on Nrf2 signaling was assessed. It was observed that calcitriol (1nM-1000nM) significantly increased nucleus Nrf2 expression compared to the control group $(P<0.01$ for the $10 \mathrm{nM}-500 \mathrm{nM}$ groups; $P<0.05$ for the $1 \mathrm{nM}$ group) (Fig. 8B, 8C). Combining the results of the above experiments, we chose 
calcitriol with $10 \mathrm{nM}$ concentration for further experiments to demonstrate the mechanism between autophagy and Nrf2 pathway in response to VitD in vitro. In order to show the autophagy flux, a tandem RFP-GFP-LC3 reporter was used. This assay takes advantage of differential pH sensitivity of GFP (acid labile) and RFP (acid resistant) fluorophores to assess acidification of autophagosomes (yellow) upon fusion with lysosomes (red only). As shown in Fig. 8D and Fig. 8E, in cells treated with calcitriol, we observed accumulation of red autolysosomes compared to controls $(P<0.01)$. However, inhibition of autophagy flux by CQ caused impairment in autophagosome-lysosome fusion, displayed an increased accumulation of yellow autophagosomes $(P<0.01)$. These data confirmed that VitD treatment induced the activation of neuronal autophagic flux. Additionally, the expression levels of autophagy-related proteins were measured by western blot. The level of p62 was higher in the calcitriol group than in the control group $(P<0.01)$. However, inhibition of autophagy flux by CQ induced the expression of LC3-II and p62 $(P<0.01)$. The expression of Keap1 was attenuated and the nuclear expression of Nrf2 was higher in the calcitriol treatment group than in control group $(P<0.01)$. However, inhibition of autophagy flux by $\mathrm{CQ}$ after calcitriol treatment markedly suppressed the Keap1-Nrf2 pathway, inducing Keap1 protein expression and decreasing Nrf2 status in the nucleus $(P<0.01)$.

\section{Discussion}

Oxidative stress is the major cause of TBI-induced secondary injury, whereas Nrf2 is an important oxidative stress regulator in the protection of various cell types and organ systems. Recent studies have also shown that impairment of Nrf2 signaling in the injured brain may aggravate ROS production and cause oxidative stress, thereby inducing inflammatory response and cell death following TBI (Zhou et al., 2018; Dong et al., 2018; Bhowmick et al., 2019). However, the pathological mechanisms remain elusive and protective strategies for the oxidative brain damage are still limited.

VitD now is recognized as a pleiotropic secosteroid affecting multiple aspects of human physiology. The beneficial effects of VitD on CNS have been observed in animal models as well as in patients with cerebral ischemia, Alzheimer's disease, Parkinson's disease, and multiple sclerosis (Cui et al., 2017; Di Somma et al., 2017). Our previous study has shown that activation of VDR is protective against neurological deficits and attenuates Nox2-induced oxidative stress in a rat model of TBI (Cui,Song 2017). In line with these findings, the present research also found that treated with calcitriol, at doses ranging from $0.5 \mu \mathrm{g} / \mathrm{kg}$ to $3 \mu \mathrm{g} / \mathrm{kg}$, was effective in potentiating the recovery process in mice following TBI by mitigating neurological deficits, improving behavioral performance in the MWM test and retarding neuronal apoptosis. These findings support the beneficial effects of VitD in TBI and further demonstrate that the sustained treatment of calcitriol immediately after TBI may enhance the recovery process and prevent potential secondary injury.

Nrf2 and autophagy are two critical stress responsive signaling pathways that are associated with redox balance. Nrf2 is a basic leucine zipper (CNC bZip) redox sensitive transcription factor that activates antioxidant response elements (AREs). The genes transcriptionally regulated by the AREs encode detoxification enzymes and antioxidant proteins thereby playing a central role in the oxidative stress 
modulation (Bhowmick,D'Mello 2019). Notably, oxidative stress and autophagy are also intricately connected. In the past few years, autophagy has been proposed as a potential survival mechanism in the context of exaggerated ROS generation by timely removal of damaged and redundant substance, serving as a cytoprotective mechanism to restrain oxidative injury (Filomeni et al., 2015). Moreover, p62dependent clearance of Keap1 has been found to regulate Nrf2 signaling and constitutes an important defense system against oxidative stress. p62 can bind and sequester Keap1, allowing the release of Nrf2 from Keap1 and Nrf2 translocation to the nucleus, thereby resulting in the activation of antioxidant genes (Dodson\Redmann 2015). As previously reported (CuīCui 2017), we found impaired autophagic flux in the injured brain with increased autophagosome accumulation. By using the lysosomal inhibitor, CQ, we confirmed the inhibited autophagic process following TBI as evidenced by the significant increase of LC3II expression in Sham group but unchanged in TBI group following CQ treatment. Although autophagy was reported to be enhanced soon after acute brain injury to cope with the stress, it seems that prolonged time after TBI is likely to induce exhaustion of autophagy (Sarkar et al., 2014). Concomitantly, we observed a marked increase of Keap1 expression and a significant decrease of Nrf2 translocation, resulting in attenuated downstream antioxidant signaling and exaggerated oxidative stress following TBI. In parallel, VitD accelerated the recovery process and activated autophagic flux following TBI. The increased transcriptional level of ATG genes but decreased protein expression of LC3-II and p62 compared with TBI group indicates that calcitriol not only induced autophagosome formation but also facilitated its degradation. Meanwhile, calcitriol also triggered Nrf2 signaling, inducing the expression of targeted genes and protecting the brain from excessive oxidative stress. In support of our findings, recent studies also illustrate a pivotal role of Nrf2 in the protective effects of VitD in Alzheimer's disease, lung injury, skin aging, liver failure and kidney toxicity (Zhang et al., 2019; Saad El-Din et al., 2020; Abo El-Magd et al., 2020; Chen et al., 2019; Tao et al., 2019). Given that Keap-1 is targeted to autophagosomes for degradation, the reduction of Keap-1 expression and the decreased co-expression of p62 and Keap1 following calcitriol treatment suggest that Nrf2 might be activated by VitD through autophagy mediated by the interaction between p62 and Keap1. By using CQ to block autophagy, we found cotreatment with CQ abrogated calcitriol-induced Keap-1 degradation and compromised Nrf2 signaling, resulting in the decreased downstream antioxidant genes and increased ROS production. As expected, both CQ treatment and Nrf2 genetic knockout abolished the beneficial effects of VitD on neurological function as well as its anti-apoptotic activity following TBI, indicating that the neuroprotective effect of VitD is through the interaction between autophagy and Nrf2 signaling. Additionally, we also observed a dose-dependent increase of Nrf2 signaling in neuronal cells following calcitriol exposure. Using the tandem fluorescent mRFP-GFP-LC3 adenovirus, we further proved that calcitriol can effectively trigger autophagic flux. Meanwhile, inhibiting autophagic process with CQ also blocked the calcitriol-induced Keap1 degradation and Nrf2 activation in vitro. These data collaboratively support the facilitating effect of calcitriol on autophagy, whereby to activate Nrf2 signaling. However, it should be noted that some researchers suggest that blocking lysosomal function would lead to autophagosome accumulation and thereby promote p62 to bind with Keap1, sequestering it from Nrf2 and resulting in Nrf2 translocation (Park et al., 2019; Menglin et al., 2018). On the other hand, there is also accumulating evidence which is in accordance with our findings, demonstrating that autophagy-induced Keap1 degradation can be 
effectively abrogated by the lysosomal inhibitors, CQ and Bafilomycin A1, which would accelerate oxidative stress or compromised the protective effects of various interventions in the brain, heart and liver tissues (Deng et al., 2020; Tan et al., 2020; Lee et al., 2020). These discrepancies might be attributed to the different cell types or disease models that may have various basal autophagic condition, and because of the different doses and treatment duration that may cause diverse autophagic inhibitory status. Following TBI or other stimuli, the injury progression and ROS production last even for weeks. To this end, the calcitriol-induced continuously autophagic clearance of Keap-1 and constantly active Nrf2 signaling are critical to mitigate the oxidative damage. In this study, CQ was co-treated with VitD, which may induce persistent inhibition on this process and thereby exacerbate brain damage.

\section{Conclusions}

Taken together, the present study firstly demonstrated that autophagic flux serves as an upstream regulator required for calcitriol-induced Nrf2 activation, thereby alleviating oxidative damage and cell apoptosis following TBI (Fig. 9). Additionally, our data demonstrated that autophagy and Nrf2, the two critical pathways in maintaining homeostasis and redox balance, are intricately interacted and both are indispensable for the antioxidant and anti-apoptosis actions of VitD. These findings would further enrich and shed novel insight on the neurological functions of VitD, providing an interesting target for the autophagic dysfunction and oxidative stress following TBI-induced neuronal damage.

\section{Abbreviations}

ATG genes, autophagy-related genes; $\mathrm{CAT}$, catalase; $\mathrm{CCl}$, controlled cortical impact; $\mathrm{CQ}$, chloroquine; $\mathrm{DHE}$, dihydroethidium; GSH, glutathione; H\&E, hematoxylin and eosin; Keap1, Kelch-like ECH-associated protein 1; MDA, Malondialdehyde; MWM, Morris water maze;Nrf2, nuclear factor E2-related factor 2; PBS, phosphate-buffered saline; ROS, reactive oxygen species; SOD, superoxide dismutase; TBI, Traumatic brain injury; TUNEL, terminal deoxynucleotidyl transferase-mediated cyanine-dUTP nick-end labeling; VDR, vitamin D receptor; VitD, vitamin D.

\section{Declarations}

\section{Acknowledgements}

We would like to thank all the colleagues in our research team.

\section{Ethics approval and consent to participate}

This study was approved by the Institutional Animal Care and Use Committee of Jining Medical University(JNMC2019DWRM007).

\section{Consent for publication}


Not applicable.

\section{Availability of data and materials}

Data are available upon reasonable request. The data used in the current study are available from the corresponding author on reasonable request.

\section{Competing interests}

The authors declare that they have no conflict of interest.

\section{Funding}

This work was supported by National Natural Science Foundation of China $(81602846,81901954)$, Natural Science Foundation of Shandong Province (No. ZR2018BH016), Taishan Scholar Project of Shandong Province (tsqn201812159), Jining Key Research and Development Program (No.

2019SMNS012), Research Fund for Lin He's Academician Workstation of New Medicine and Clinical Translation of Jining Medical University (JYHL2018FZD01), Shandong Medical and Health Science and Technology Development Program Project (2017WS511).

\section{Author contributions}

Conceptualization, P.J., C.C. and C.W. ; methodology, F.J. and P.J.; software, C.C. and C.W.; validation, M.Y., L.K. and W.H.; formal analysis, C.W.; investigation, M.Y., L.K. and W.H.; resources, P.J. and C.C; data curation, C.C. and C.W. ; writing-Original draft preparation, C.C. and C.W.; writing-Review and editing, P.J. and C.C.; visualization, C.C. and C.W.; supervision, P.J. and C.W. ; project administration, P.J. All authors have read and agreed to the published version of the manuscript.

\section{References}

1. Stout DM, Buchsbaum MS, Spadoni AD, Risbrough VB, Strigo IA, Matthews SC, et al. Multimodal canonical correlation reveals converging neural circuitry across trauma-related disorders of affect and cognition. Neurobiology of stress. 2018;9:241-50.

2. Ma MW, Wang J, Dhandapani KM, Wang R, Brann DW. NADPH oxidases in traumatic brain injury Promising therapeutic targets? Redox Biol. 2018;16:285-93.

3. Zhang Z, Zhang L, Zhou L, Lei Y, Zhang Y, Huang C. Redox signaling and unfolded protein response coordinate cell fate decisions under ER stress. Redox Biol. 2019;25:101047.

4. Su SH, Wu YF, Lin Q, Wang DP, Hai J. URB597 protects against NLRP3 inflammasome activation by inhibiting autophagy dysfunction in a rat model of chronic cerebral hypoperfusion. J Neuroinflamm. 2019;16(1):260.

5. Saito Y, Yako T, Otsu W, Nakamura S, Inoue Y, Muramatsu A, et al. A triterpenoid Nrf2 activator, RS9, promotes LC3-associated phagocytosis of photoreceptor outer segments in a p62-independent 
manner. Free Radic Biol Med. 2020;152:235-47.

6. Zheng D, Liu Z, Zhou Y, Hou N, Yan W, Qin Y, et al. Urolithin B, a gut microbiota metabolite, protects against myocardial ischemia/reperfusion injury via p62/Keap1/Nrf2 signaling pathway. Pharmacological research. 2020;153:104655.

7. Deng S, Essandoh K, Wang X, Li Y, Huang W, Chen J, et al. Tsg101 positively regulates P62-Keap1Nrf2 pathway to protect hearts against oxidative damage. Redox biology. 2020:101453.

8. Dodson M, Redmann M, Rajasekaran NS, Darley-Usmar V, Zhang J. KEAP1-NRF2 signalling and autophagy in protection against oxidative and reductive proteotoxicity. Biochem J. 2015;469(3):34755.

9. Cui C, Xu P, Li G, Qiao Y, Han W, Geng C, et al. Vitamin D receptor activation regulates microglia polarization and oxidative stress in spontaneously hypertensive rats and angiotensin II-exposed microglial cells: Role of renin-angiotensin system. Redox Biol. 2019;26:101295.

10. Cui C, Song S, Cui J, Feng Y, Gao J, Jiang P. Vitamin D Receptor Activation Influences NADPH Oxidase (NOX2) Activity and Protects against Neurological Deficits and Apoptosis in a Rat Model of Traumatic Brain Injury. Oxidative medicine and cellular longevity. 2017;2017:9245702.

11. Cui C, Cui J, Jin F, Cui Y, Li R, Jiang X, et al. Induction of the Vitamin D Receptor Attenuates Autophagy Dysfunction-Mediated Cell Death Following Traumatic Brain Injury. Cellular physiology and biochemistry: international journal of experimental cellular physiology, biochemistry, and pharmacology. 2017;42(5):1888-96.

12. Zheng J, Liu X, Zheng B, Zheng Z, Zhang H, Zheng J, et al. Maternal 25-Hydroxyvitamin D Deficiency Promoted Metabolic Syndrome and Downregulated Nrf2/CBR1 Pathway in Offspring. Front Pharmacol. 2020;11:97.

13. Nachliely M, Trachtenberg A, Khalfin B, Nalbandyan K, Cohen-Lahav M, Yasuda K, et al. Dimethyl fumarate and vitamin D derivatives cooperatively enhance VDR and Nrf2 signaling in differentiating AML cells in vitro and inhibit leukemia progression in a xenograft mouse model. J Steroid Biochem Mol Biol. 2019;188:8-16.

14. Zhou Y, Wang HD, Zhou XM, Fang J, Zhu L, Ding K. N-acetylcysteine amide provides neuroprotection via Nrf2-ARE pathway in a mouse model of traumatic brain injury. Drug Des Devel Ther. 2018;12:4117-27.

15. Dong W, Yang B, Wang L, Li B, Guo X, Zhang M, et al. Curcumin plays neuroprotective roles against traumatic brain injury partly via Nrf2 signaling. Toxicol Appl Pharmcol. 2018;346:28-36.

16. Bhowmick S, D'Mello V, Caruso D, Abdul-Muneer PM. Traumatic brain injury-induced downregulation of Nrf2 activates inflammatory response and apoptotic cell death. Journal of molecular medicine. 2019;97(12):1627-41.

17. Cui X, Gooch H, Petty A, McGrath JJ, Eyles D. Vitamin D and the brain: Genomic and non-genomic actions. Molecular cellular endocrinology. 2017;453:131-43.

18. Di Somma C, Scarano E, Barrea L, Zhukouskaya VV, Savastano S, Mele C, et al. Vitamin D and Neurological Diseases: An Endocrine View. International journal of molecular sciences. 2017;18(11). 
19. Filomeni G, De Zio D, Cecconi F. Oxidative stress and autophagy: the clash between damage and metabolic needs. Cell death differentiation. 2015;22(3):377-88.

20. Sarkar C, Zhao Z, Aungst S, Sabirzhanov B, Faden Al, Lipinski MM. Impaired autophagy flux is associated with neuronal cell death after traumatic brain injury. Autophagy. 2014;10(12):2208-22.

21. Zhang H, Xue L, Li B, Zhang Z, Tao S. Vitamin D. Protects Against Alcohol-Induced Liver Cell Injury Within an NRF2-ALDH2 Feedback Loop. Molecular nutrition food research. 2019;63(6):e1801014.

22. Saad El-Din S, Rashed L, Medhat E, Emad Aboulhoda B, Desoky Badawy A, Mohammed ShamsEldeen $A$, et al. Active form of vitamin $D$ analogue mitigates neurodegenerative changes in Alzheimer's disease in rats by targeting Keap1/Nrf2 and MAPK-38p/ERK signaling pathways. Steroids. 2020;156:108586.

23. Abo El-Magd NF, Eraky SM. The molecular mechanism underlining the preventive effect of vitamin $D$ against hepatic and renal acute toxicity through the NrF2/ BACH1/ HO-1 pathway. Life sciences. 2020;244:117331.

24. Chen L, Yang R, Qiao W, Zhang W, Chen J, Mao L, et al. 1,25-Dihydroxyvitamin D exerts an antiaging role by activation of Nrf2-antioxidant signaling and inactivation of p16/p53-senescence signaling. Aging cell. 2019;18(3):e12951.

25. Tao $S$, Zhang $H$, Xue L, Jiang $X$, Wang $H$, Li B, et al. Vitamin D protects against particles-caused lung injury through induction of autophagy in an Nrf2-dependent manner. Environmental toxicology. 2019;34(5):594-609.

26. Park JY, Kim S, Sohn HY, Koh YH, Jo CJSR. TFEB activates Nrf2 by repressing its E3 ubiquitin ligase DCAF11 and promoting phosphorylation of p62. 2019;9(1).

27. Menglin F, Yanan, Chunhua, Yao X, et al. DC32, a Dihydroartemisinin Derivative, Ameliorates Collagen-Induced Arthritis Through an Nrf2-p62-Keap1 Feedback Loop. 2018.

28. Deng S, Essandoh K, Wang X, Li Y, Fan GCJRB. Tsg101 positively regulates P62-Keap1-Nrf2 pathway to protect hearts against oxidative damage. 2020;32:101453.

29. Tan X, Yang Y, Xu J, Zhang P, Chen GJFiP. Luteolin Exerts Neuroprotection via Modulation of the p62/Keap1/Nrf2 Pathway in Intracerebral Hemorrhage. 2020;10:1551.

30. Lee DH, Park JS, Lee YS, Han J, Lee DK, Kwon SW, et al. SQSTM1/p62 activates NFE2L2/NRF2 via ULK1-mediated autophagic KEAP1 degradation and protects mouse liver from lipotoxicity. 2020(1).

\section{Figures}


A

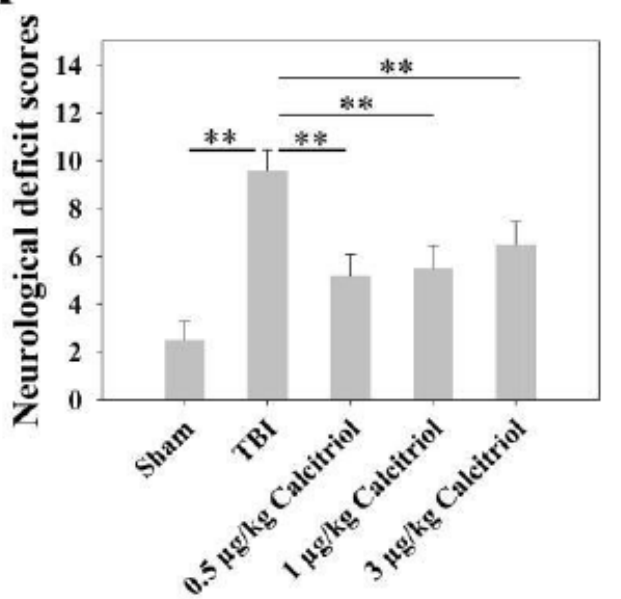

B

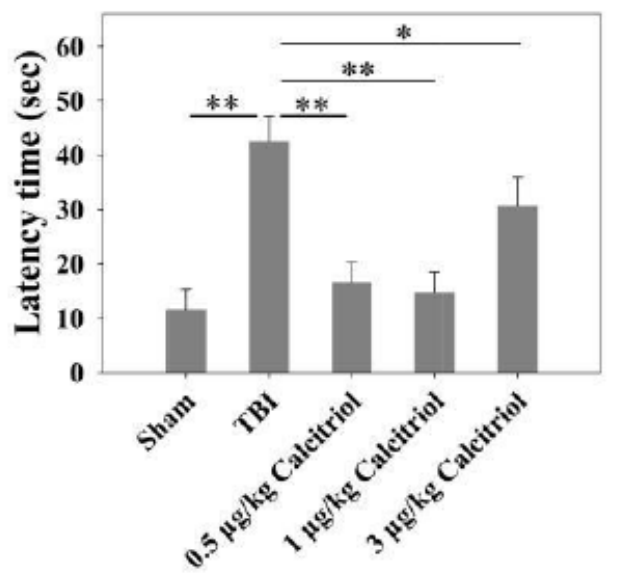

C

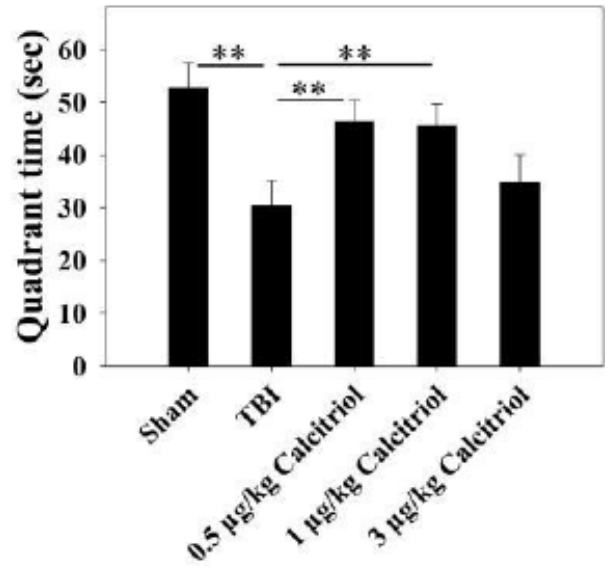

D
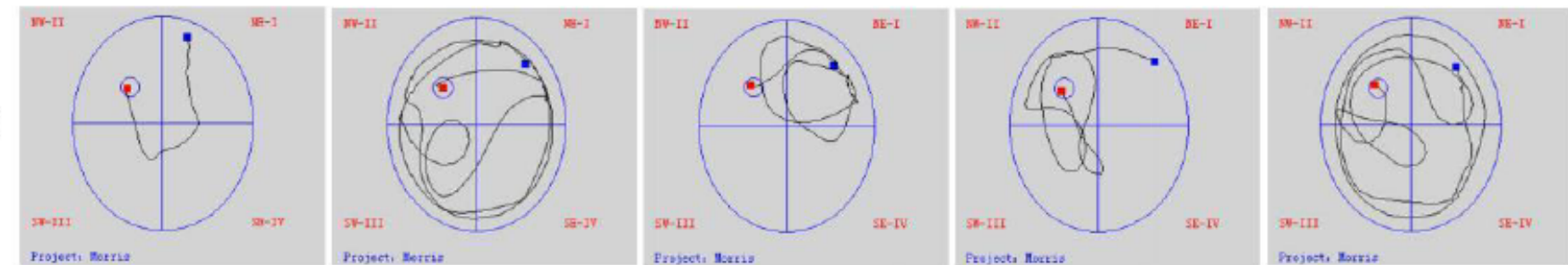

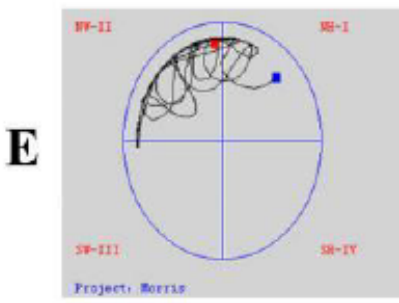

Sham

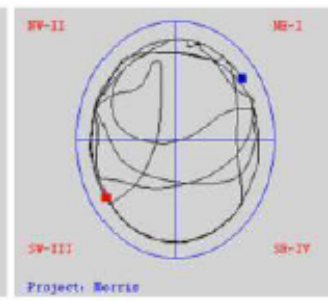

TBI

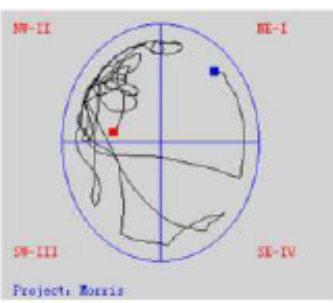

$0.5 \mu \mathrm{g} / \mathrm{kg}$ Calcitriol

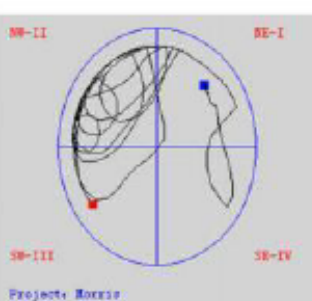

$1 \mu \mathrm{g} / \mathrm{kg}$ Calcitriol

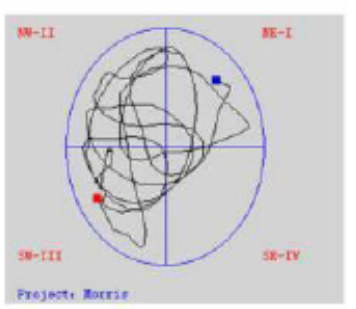

$3 \mu \mathrm{g} / \mathrm{kg}$ Calcitriol

\section{Figure 1}

The neuroprotective effects of calcitriol in TBI-induced neurological deficits and memory dysfunction. A, The variation in neurological deficits at 14 days after treatment was determined by neurological severity score tests. B, The time (seconds) spent finding the submerged platform at 14 days. C, The time (seconds) spent on exploring the quadrant that initially contained the platform at 14 days. D, Representative traces indicating the sample paths of the mice in the probe trials. E, Representative traces indicating the sample paths of the mice after the platform was removed. Data are presented as means \pm $S D(n=10) .{ }^{*} P<0.05$ and ${ }^{*} P<0.01$ versus the indicated groups. 


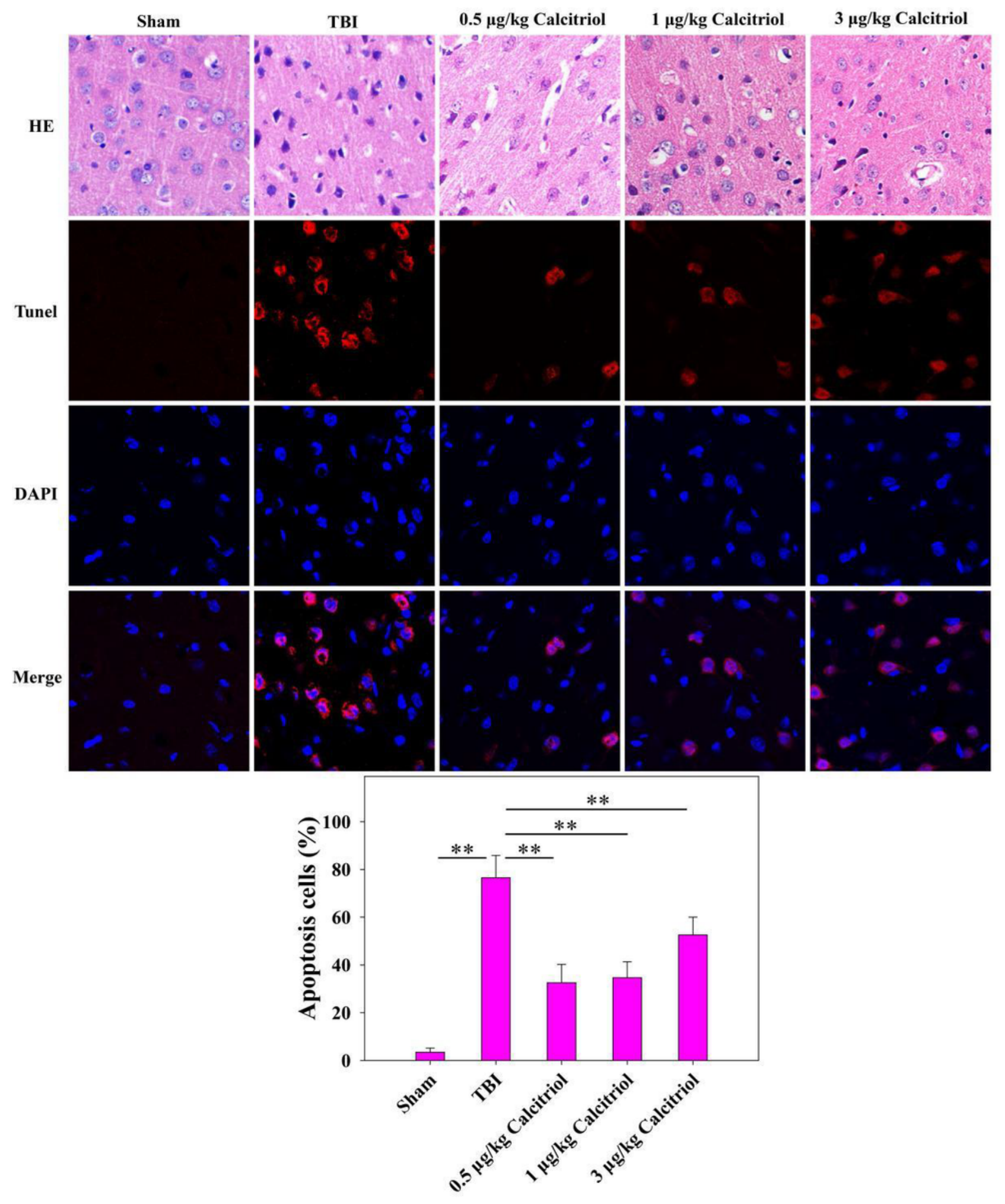

Figure 2

Calcitriol treatment mitigated TBI-induced histopathological changes. A, Representative images of the histological assessment of the frontal cortex via hematoxylin and eosin (H\&E) and TUNEL staining $(\times 400)$. B, Statistical graphs of apoptosis cells (\% of DAPI). Data are presented as means \pm SD $(n=5)$. ${ }^{\star * P}$ $<0.01$ versus the indicated groups. 
A

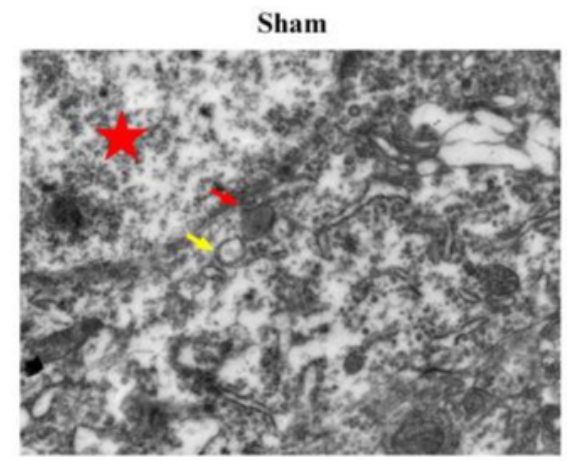

TBI
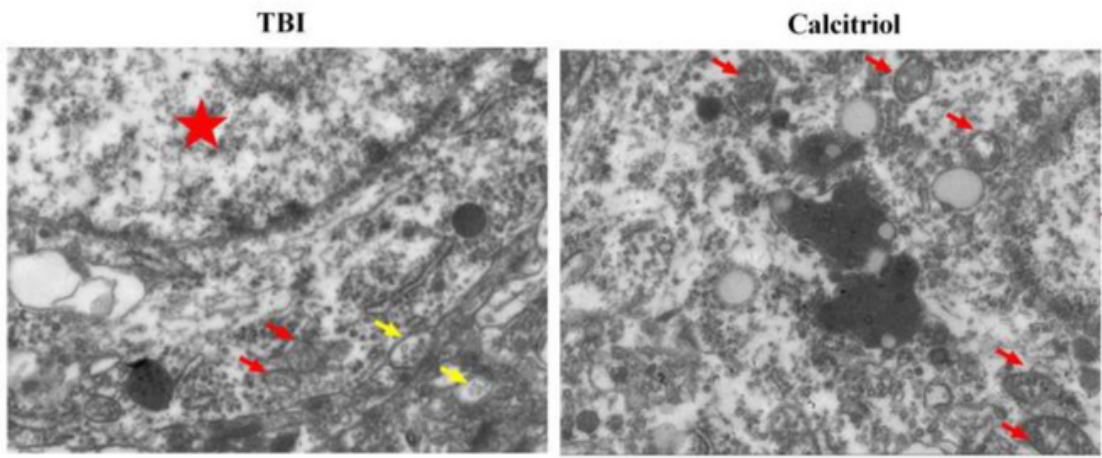

B

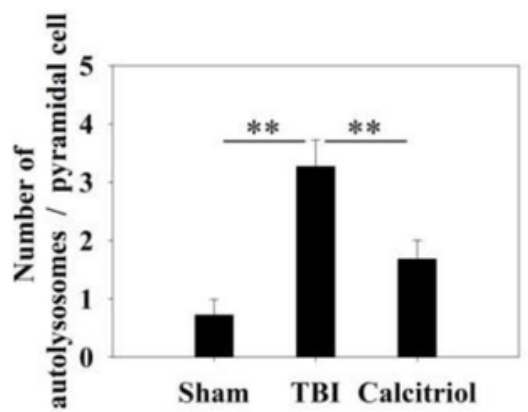

C
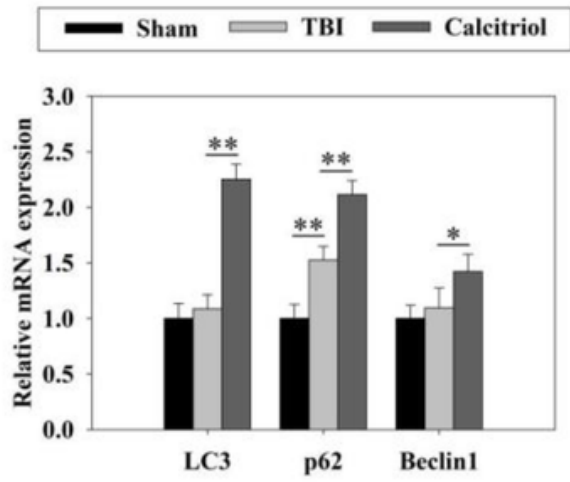

D

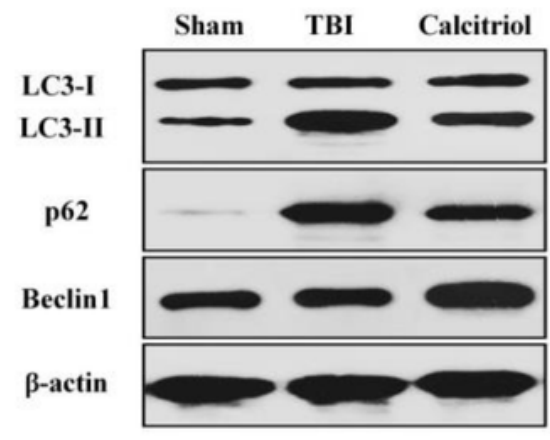

E
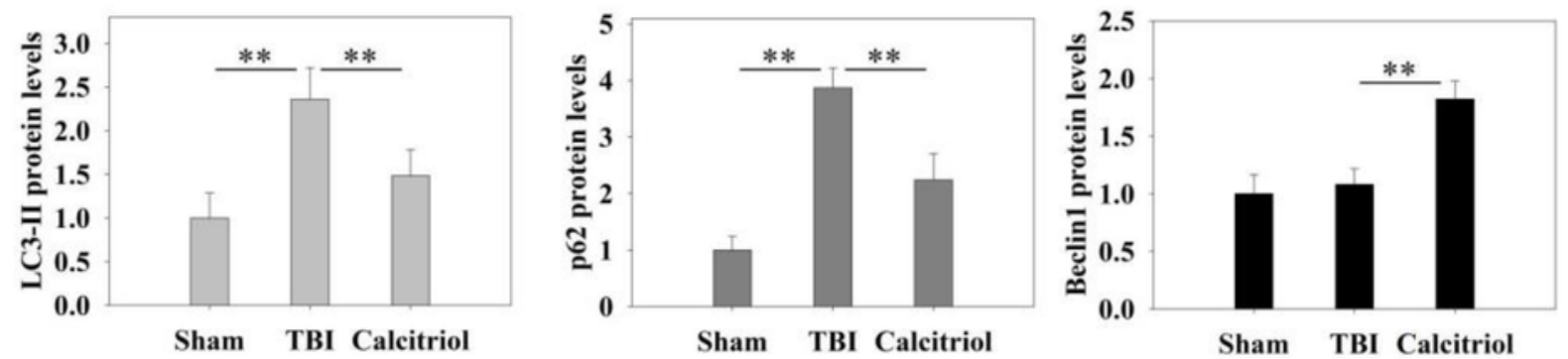

F

G
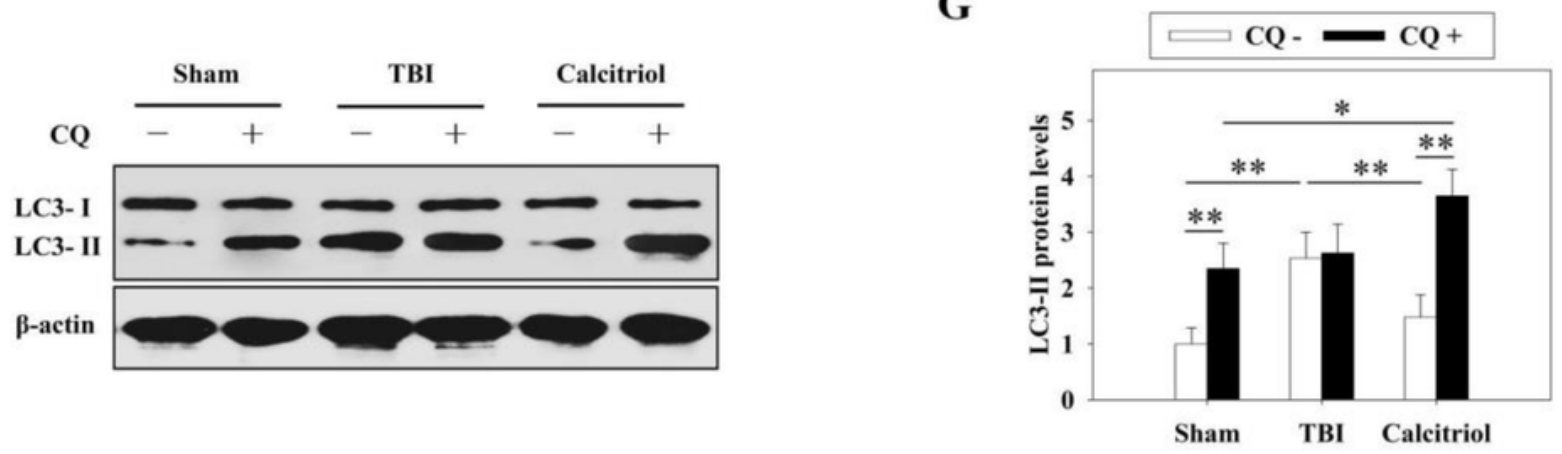

\section{Figure 3}

Calcitriol treatment attenuated TBl-induced autophagic dysfunction. A, Representative transmission electron microscopy (TEM) images of 3 groups. Red pentagram denote representative nucleus, yellow arrows denote representative autophagosomes and red arrows denote representative autolysosomes. B, Statistical graphs of number of autophagosomes and autolysosomes per cell. C, The effects of calcitriol treatment and TBI on the mRNA expression levels of autophagic markers. D, Representative images of 
western blots of autophagic markers. E, Graphs of LC3-II, p62, and beclin 1 protein expression levels. F, G, Chloroquine (CQ) was used to evaluate the effects of calcitriol treatment and TBI on autophagic flux. Data are presented as means $\pm S D(n=5) . * P<0.05$ and $* * P<0.01$ versus the indicated groups.

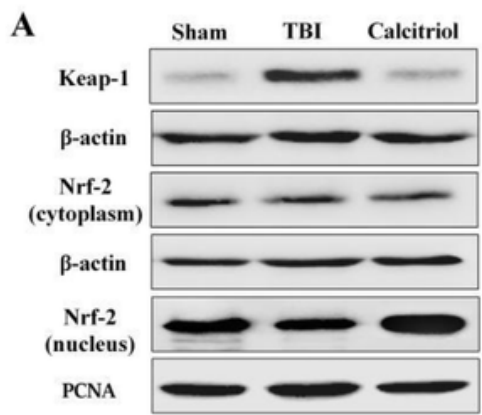

C
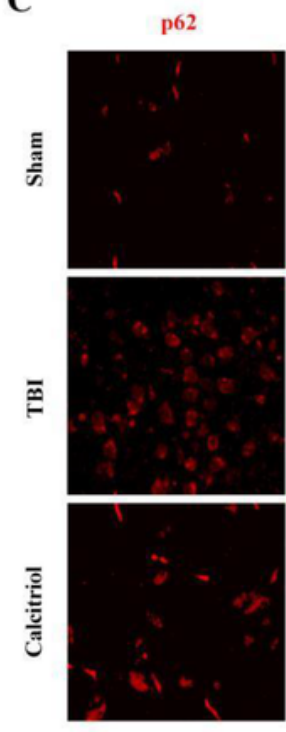

$\mathbf{E}$

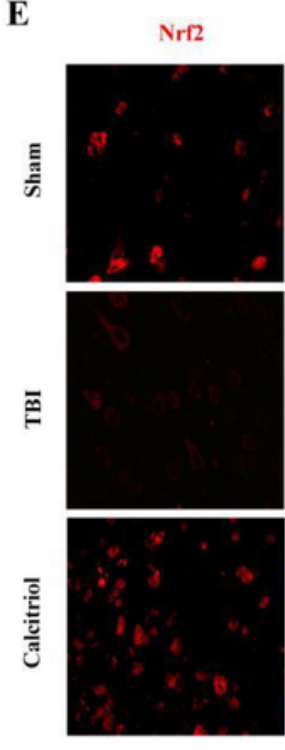

Keap-1
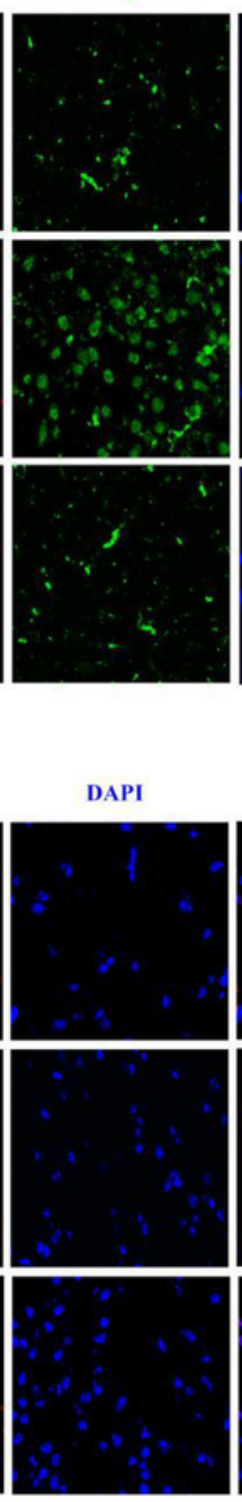

B
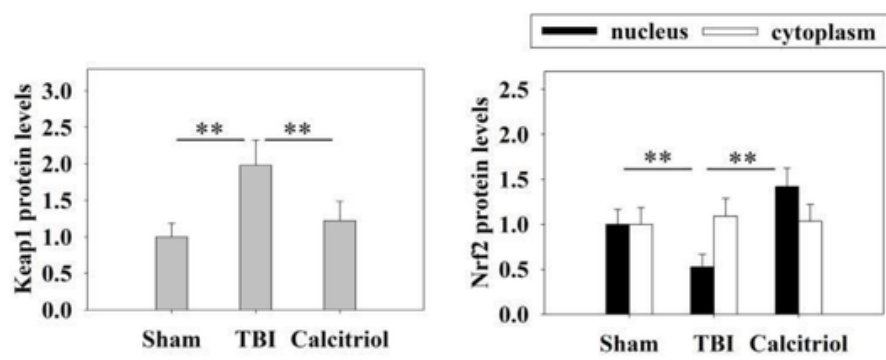

D
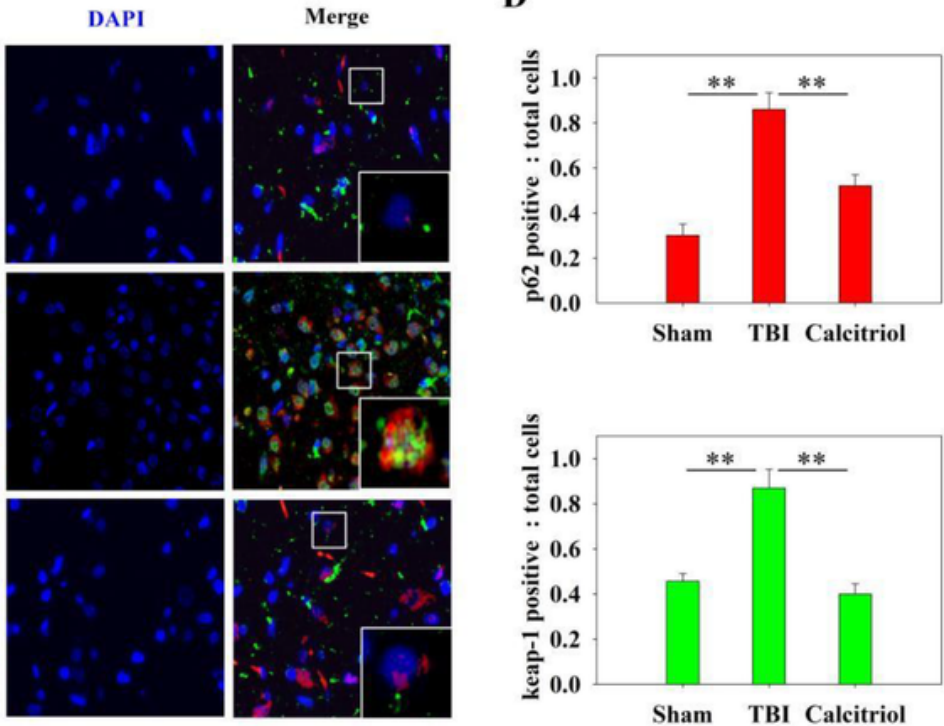

G

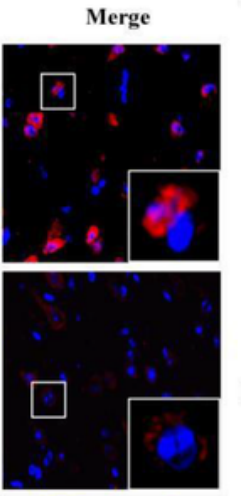

F

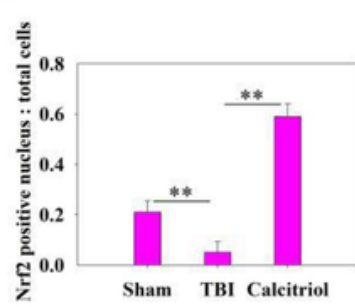

$\mathbf{H}$

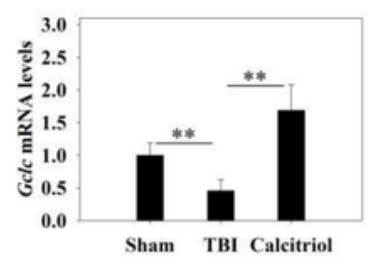

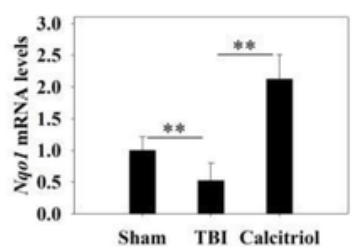

I

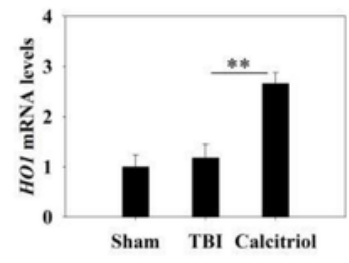

Figure 4

The effects of calcitriol on Nrf2 signaling after TBI. A, Representative images of western blot staining for Keap1, cytoplasmic Nrf2, and nuclear Nrf2. B, Statistical graphs of Keap1 protein expression and Nrf2 
translocation. C, D, Representative immunofluorescence images and statistical graphs of p62 and Keap1 co-expression. E, F, Representative immunofluorescence images and statistical graphs of Nrf2 translocation. G, The relative mRNA expression level of Nqo1. $\mathrm{H}$, The relative mRNA expression level of Gclc. I, The relative mRNA expression level of HO1. Data are presented as means $\pm S D(n=5)$. ${ }^{*}<0.05$ and $* * P<0.01$ versus the indicated groups.

A

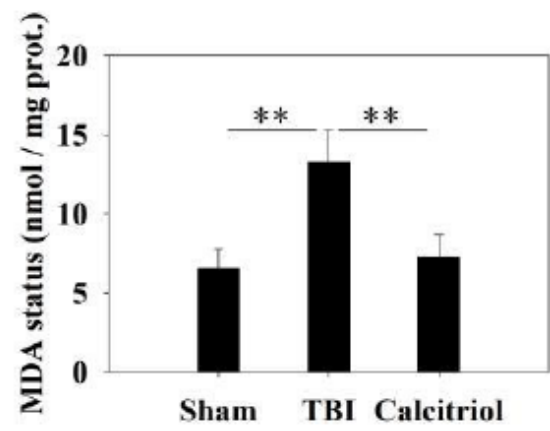

D

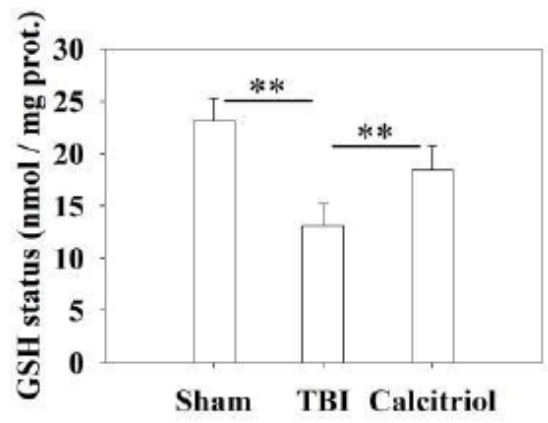

$\mathbf{F}$

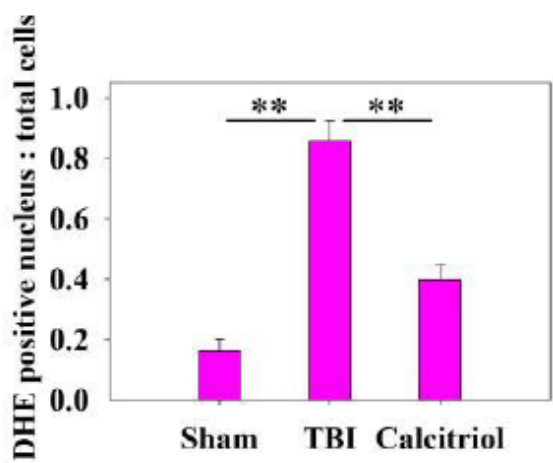

B

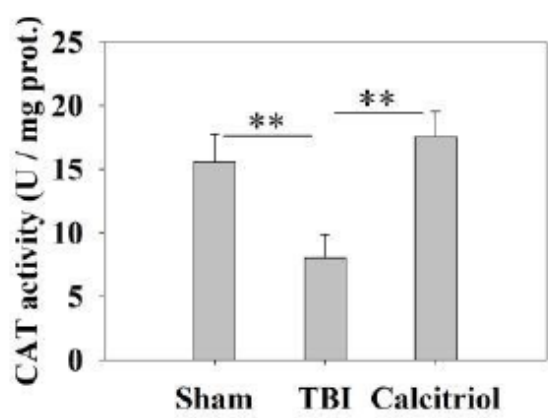

$\mathbf{E}$
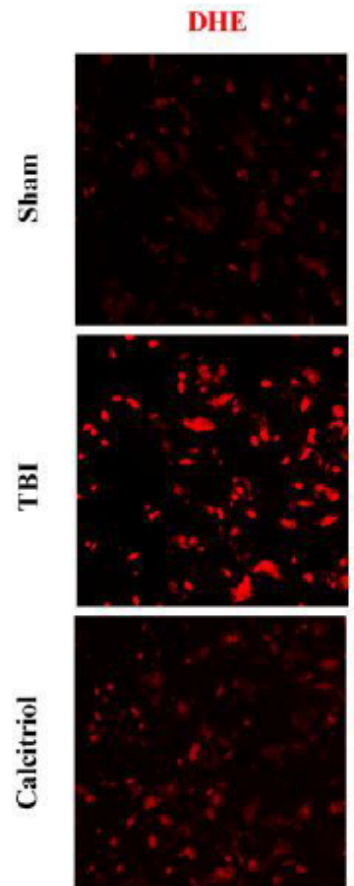
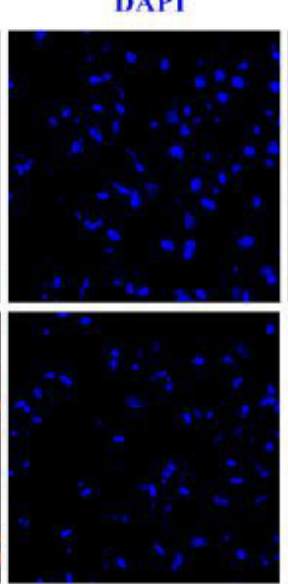

C

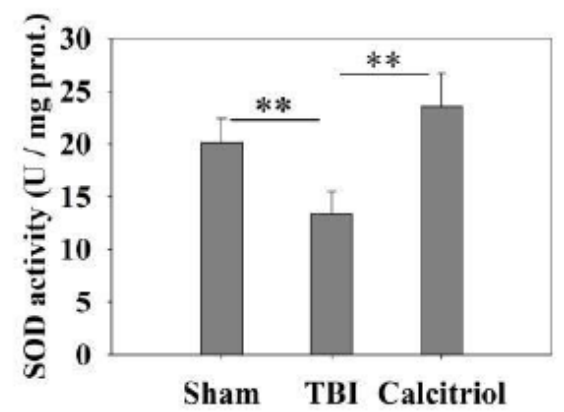

Sham TBI Calcitriol

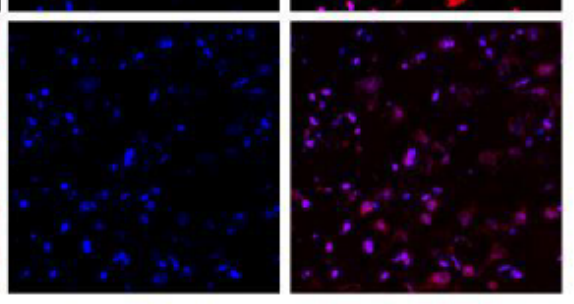

\section{Figure 5}

Calcitriol treatment ameliorated TBI-induced oxidative stress. A, Malondialdehyde (MDA) status. B, Catalase (CAT) activity. C, Superoxide dismutase (SOD) activity. D, Glutathione (GSH) status. E, Representative images of immunofluorescence assays of dihydroethidium (DHE). F, Statistical graphs of DHE-positive cells in different groups. Data are presented as means $\pm S D(n=5) .{ }^{*} P<0.01$ versus the indicated groups. 
A

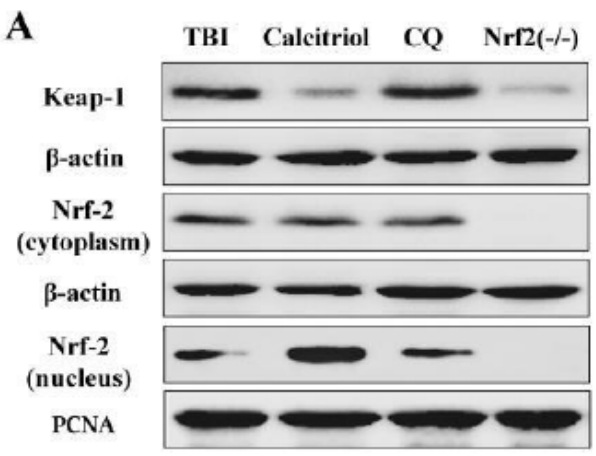

D

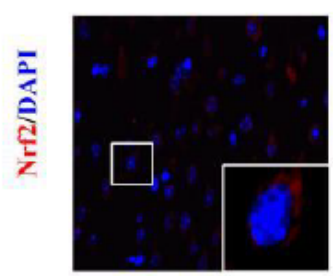

TBI

$\mathbf{F}$

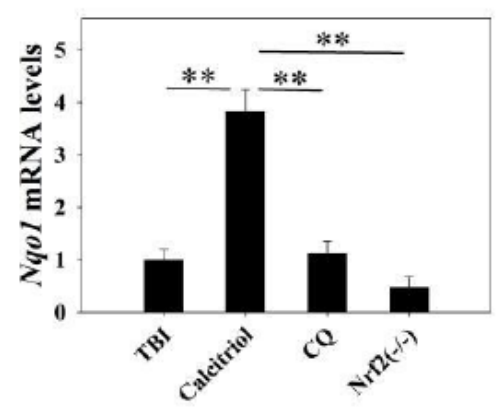

I

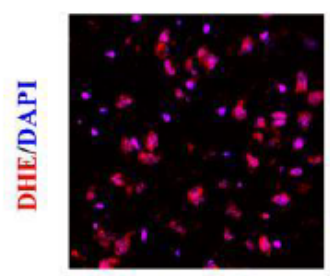

TBI

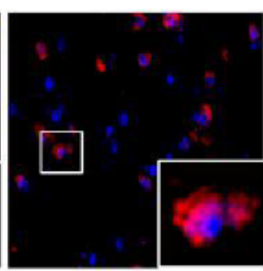

Calcitriol

G

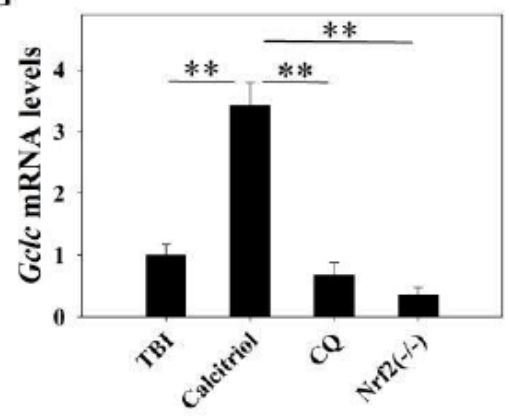

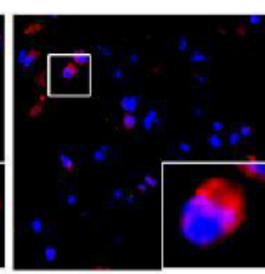

CQ

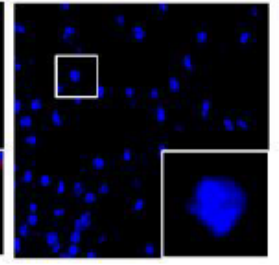

$\operatorname{Nrf2(-/-)}$
C

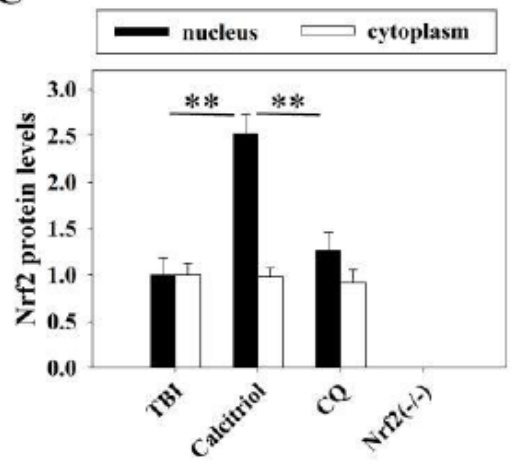

$\mathrm{E}$

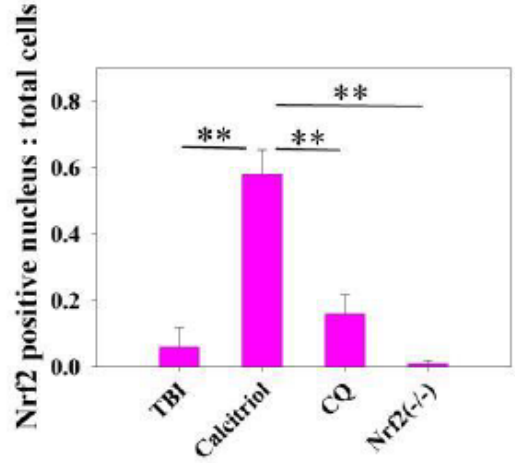

H

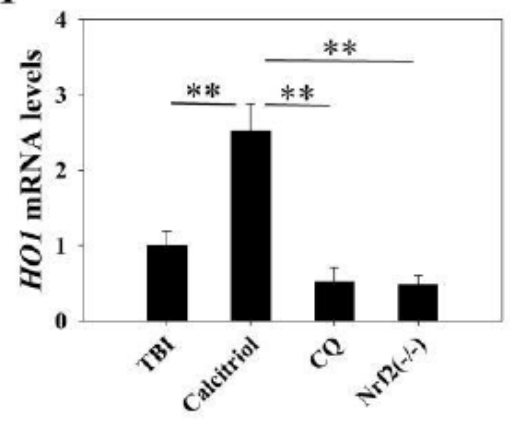

$\mathbf{J}$

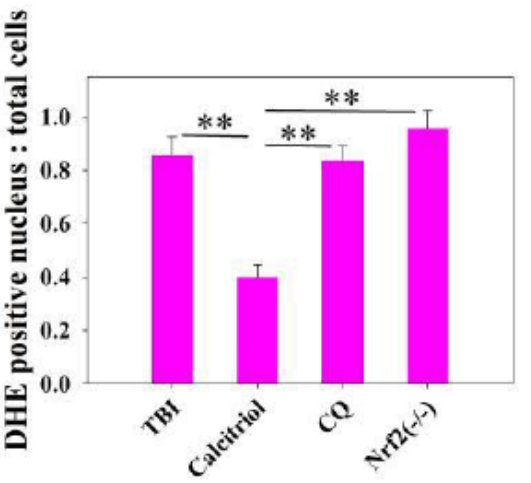

\section{Figure 6}

Inhibiting autophagy abolished calcitriol-induced Nrf2 activation following TBI. A, Representative images of western blot staining for Keap1, cytoplasmic Nrf2, and nuclear Nrf2. B, Statistical graphs of Keap1 protein expression. C, Statistical graphs of expression of Nrf2 in the nucleus and cytoplasm. D, E, Representative immunofluorescence images and statistical graphs of Nrf2 translocation. F, The relative mRNA expression level of Nq01. G, The relative mRNA expression level of Gclc. $\mathrm{H}$, The relative mRNA 
expression level of H01. I, J, Representative images and statistical graphs of immunofluorescence assays of dihydroethidium (DHE). Data are presented as means $\pm S D(n=5) .{ }^{*} P<0.05$ and ${ }^{* * P}<0.01$ versus the indicated groups.
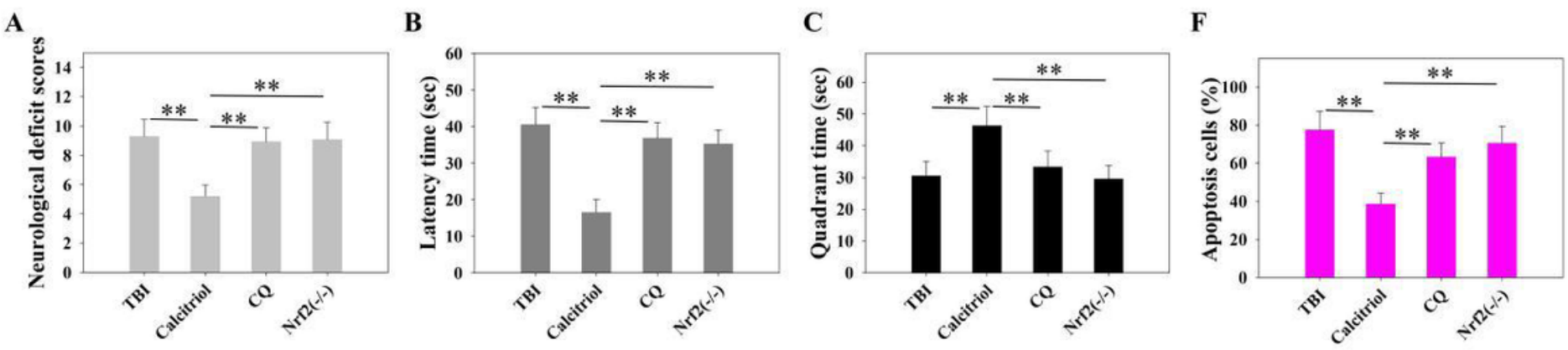

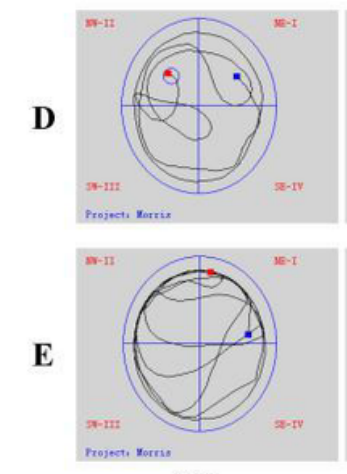

TBI
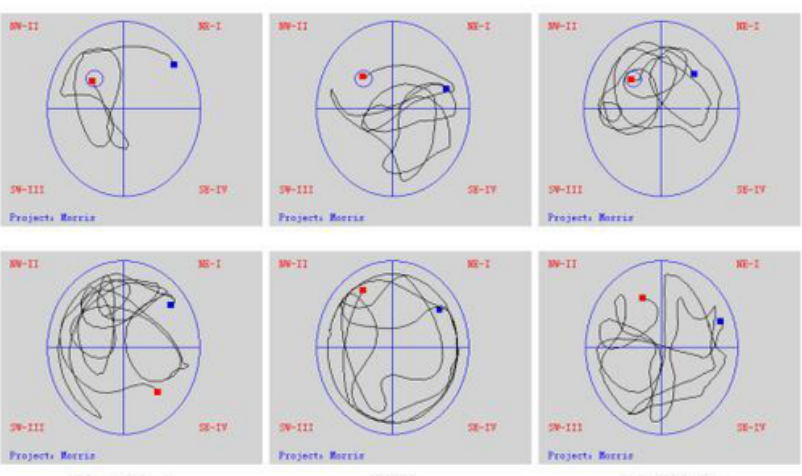

Calcitriol

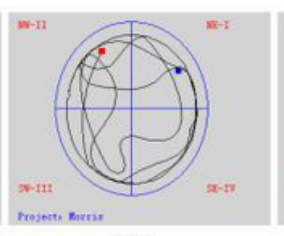

CQ

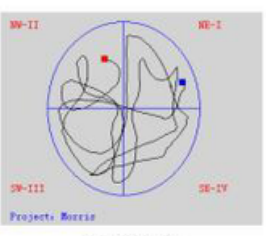

Nrf2 (-/-)

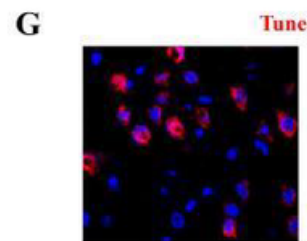

TBI

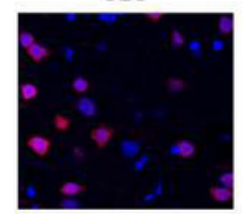

CQ

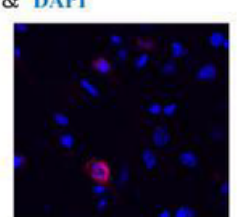

Calcitriol

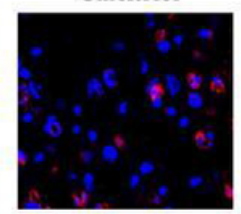

$\operatorname{Nrf2}(-/-)$

\section{Figure 7}

Blocking Nrf2 and autophagy abrogated the protective effects of calcitriol on TBI-induced neurological dysfunction. A, The variation in neurological deficits at 14 days after treatment was determined by neurological severity score tests. B, The time (seconds) spent finding the submerged platform at 14 days. C, The time (seconds) spent exploring the quadrant that initially contained the platform at 14 days. D, Representative traces from the probe trials indicating the sample paths of the mice. E, Representative traces after the platform was removed indicating the sample paths of the mice. F, Statistical graphs of apoptotic cells (\% of DAPI). G, Representative immunofluorescence assays of TUNEL-positive cells. Data are presented as means $\pm S D(n=10) . * P<0.05$ and ${ }^{*} P<0.01$ versus the indicated groups. 
A

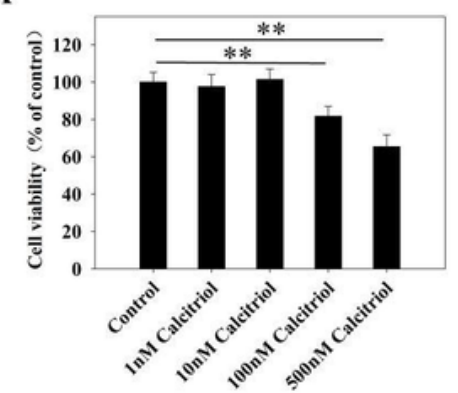

C

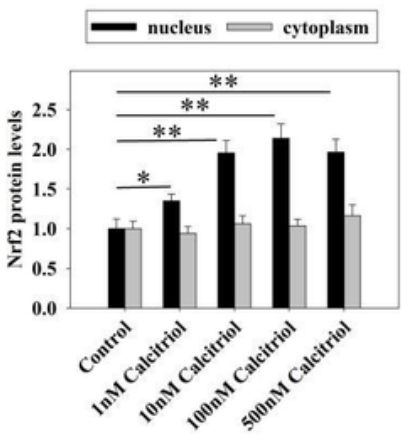

$\mathbf{E}$

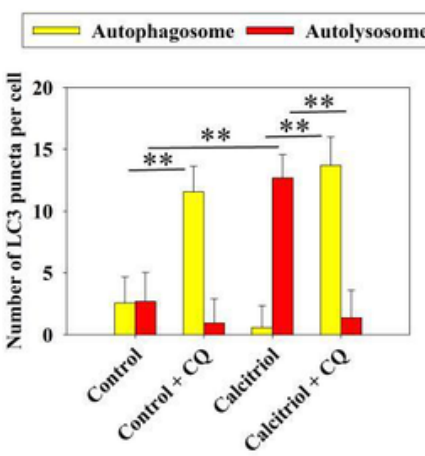

$\mathbf{F}$
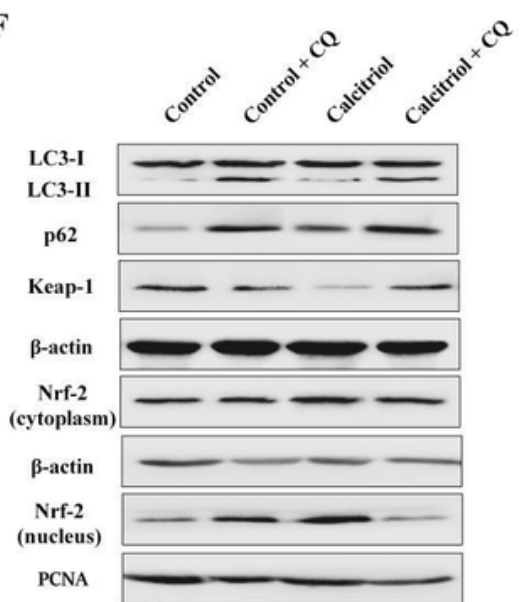

B

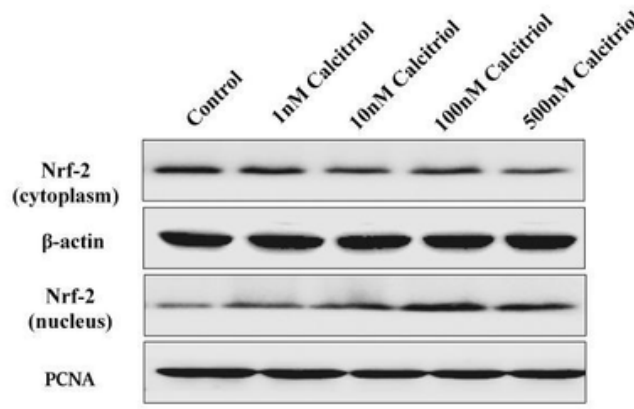

D

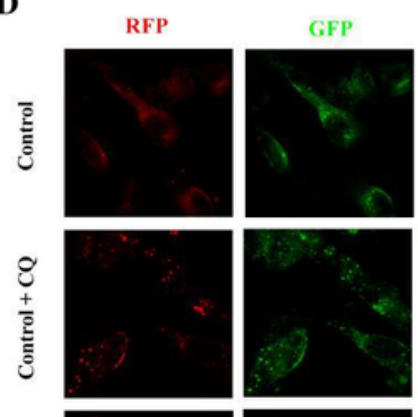

DAPI
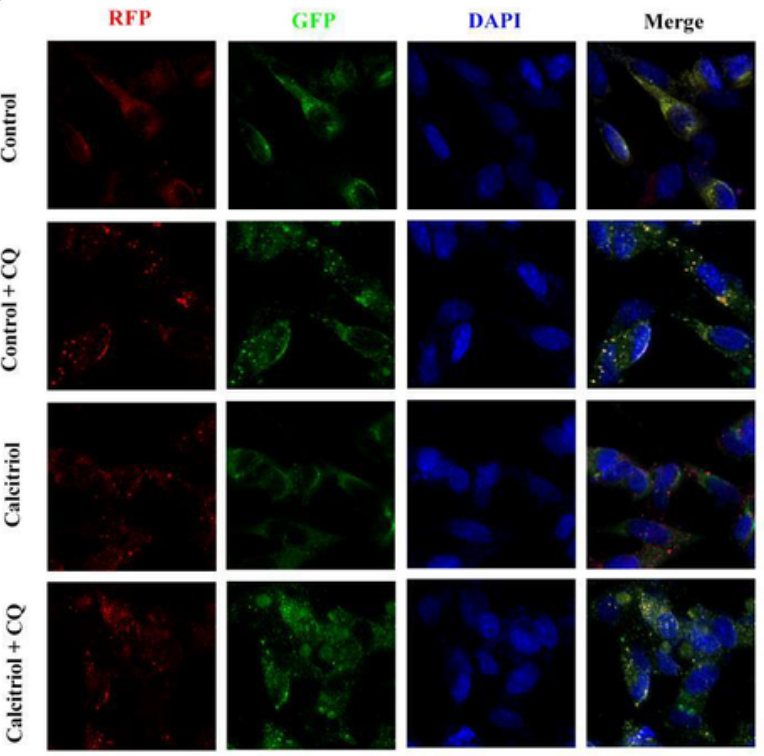

G
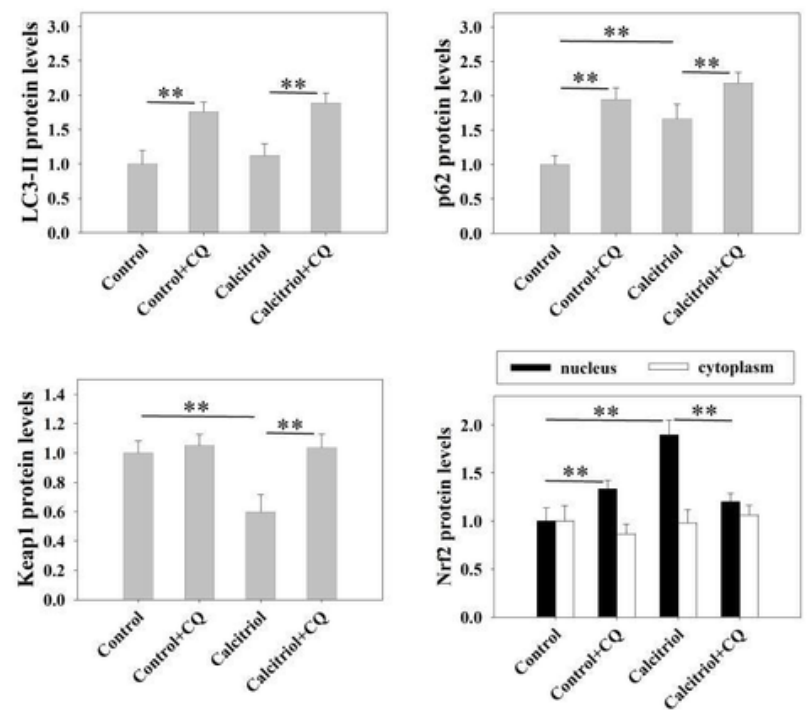

\section{Figure 8}

Effect of calcitriol on neuronal autophagy and Keap1-Nrf2 pathway in vitro. A, Statistical graphs of the variation in cell viability detected by MTT assay. B, C, Representative images and statistical graphs of western blot staining for cytoplasmic Nrf2 and nuclear Nrf2 after different dosages of calcitriol treatment. D, Representative immunofluorescence images of autophagy flux detected by a tandem RFP-GFP-LC3 reporter. E, Statistical graphs of number of autophagosomes and autolysosomes per cell. F, 
Representative images of western blot staining for LC3, p62, Keap1, cytoplasmic Nrf2, and nuclear Nrf2. G, Statistical graphs of LC3-II, p62, Keap1 and Nrf2 (cytoplasmic and nuclear) protein expression levels. Data are presented as means $\pm S D(n=5)$. ${ }^{*} P<0.05$ and ${ }^{*} * P<0.01$ versus the indicated groups.

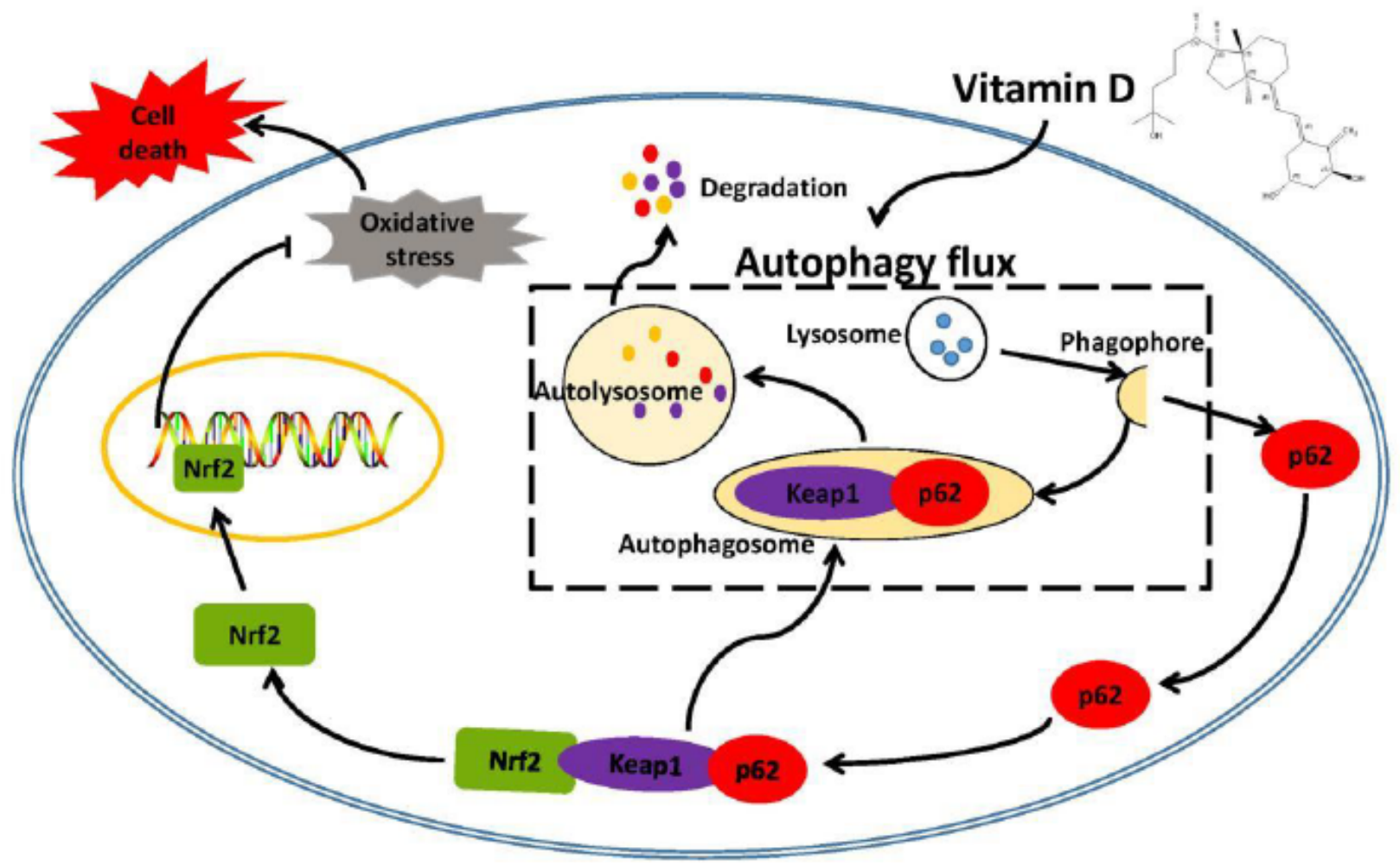

Figure 9

The molecular mechanisms underlying the neuroprotective effects of VitD against TBI. VitD maintains redox balance and protects the brain from oxidative damage through Nrf2 signaling mediated by the autophagic degradation of Keap1.

\section{Supplementary Files}

This is a list of supplementary files associated with this preprint. Click to download.

- Additionalfile1.docx

- Additionalfile2.docx

- Additionalfile3.tif

- Additionalfile4.tif

- Additionalfile5.tif

- Additionalfile6.tif 